\title{
Ueber den syphilitischen Primäraffekt am Nabel.
}

\author{
Von \\ Prof. Dr. Rille, \\ Direktor der dermatologisehen Klinik an der Universität Loipzig.
}

Von den syphilitischen Primäraffekten der Rumpfhaut haben die der Brustdrüsengegend aus ätiologischen und prophylaktischen Gründen von jeher am meisten interessiert. Große praktische Bedeutung kommt auch den bereits "perigenital" lokalisierten, nicht seltenen und meist durch größere Dimensionen ausgezeichneten Sklerosen der Unterbauchgegend und des Mons Veneris zu. Fast gar keine Beachtung fand dagegen - wohl wegen ihrer exzeptionellen Seltenheit - die an der Nabelnarbe situierte Primäraffektion.

Die älteste Beobachtung stammt von Bleynie, 1 ) Prof. der Geburtshilfe in Limoges. Dieser berichtet von einer 1855 in der kleinen Arrondissements-Hauptstadt Rochechouart (Departement Haute-Vienne) herrschenden Syphilisendemie. Die dort tätige Hebamme hatte die Gewohnheit, bei den ihrer Obhut anvertrauten Neugeborenen die nach Ablation des Nabelstranges restierende kleine Wunde mit zweien ihrer vorher mit Speichel befeuchteten Finger zu massieren. Da sie mit Syphilis behaftet war, so warde eine große Zahl von Kindern in der Nabelgegend infiziert. Nähere Angaben über

1) Bleynie, bei Bardinet, Syphilis communiquée par le doigt d'une sage-femme à un grand nombre d'accouchées de la Ville de Brive. Bullet. de l'Acad. de Méd. 37. Jahrg., Paris 1874, p. 319.

Archiv f. Dermat, u. Syph, Bd. CXIII. 
den klinischen Befund und den weiteren Verlauf sind leider nicht vorhanden.

Eine weitere, gleichfalls ein kleines Kind betreffende Syphilisinfektion am Nabel hat $\mathrm{Neumann} \mathrm{n}^{\mathbf{1}}$ ) verzeichnet. Ein Handlungsreisender, der sich unterwegs einen syphilitischen Primäraffekt zugezogen hatte, infizierte seine im 7. Monate gravide Gattin, bei welcher sich dann im 8 . Schwangerschaftsmonate eine Sklerose entwickelte: Das Kind kam zwar reif und syphilisfrei zur Welt, doch akquirierte es im Alter von 5 Monaten von seiner mit breiten Kondylomen der Genital- und Afterregion behafteten Mutter, bei der es im selben Bette lag, einen Primäraffekt am Nabel und ein sich daran anschließendes papulopustulöses Syphilid.

Nicht hierher gehörig ist eine weitere, ebenfalls an einem Kinde gemachte Beobachtung von Wolbarst, ${ }^{9}$ ) welche in

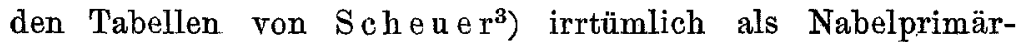
affekt geführt wird. Bei diesem 3jährigen, von syphilisfreien Eltern stammenden, im übrigen mit gonorrhoischer Urethritis posterior, linksseitiger Epididymitis und Vergrößerung der Prostata behafteten Knaben saß jedoch der Primäraffekt einen Zoll (= 2.5 cm) unterhalb des Nabels.

Leloir ${ }^{4}$ beschreibt einen Fall von primärer Nabelsyphilis, welcher übrigens von $\mathrm{Bulkle \textrm {y } ^ { 5 }}$ ) in seiner großen Statistik der extragenitalen Lues übersehen worden ist. Ein an Primäraffekt des Penis leidender verheirateter Mann fest überzengt, die Übertragung der Syphilis auf seine Gattin auf diese Art verhüten zu können - hatte die Kohabitation versus umbilicum feminae ausgeführt, was bei dem Embonpoint

1) Neumann. Über extragenit. syphil, Primäraffekte. Internat. klin. Rundschau. 1892. p. 603.

- Über ungewöhnlichen Sitz des Primäraffektes an der Haut und Schleimhaut. Wiener med. Presse. Bd. XLIII. 1902. p. 403 und 406.

2) Wolbarst. Extragenital chancres. New-York Med. Journ., Bd. LXXXII. 1905. p. 436.

$\left.{ }^{8}\right)$ Scheuer. Die Syphilis der Unschuldigen. Berlin und Wien 1910. p. 147.

4) Leloir. Leçons sur la Syphilis. Paris 1886. p. 45.

5) Bulkley. Syphilis in the innocent. New.York 1894. 
der Frau ganz gut gelungen sein soll. Die Folge war primäre Syphilis der Nabelgegend nebst Konsekutiverscheinungen.

Eine ganz ähnliche Beobachtung machte Fournier1) bei einer gleichfalls wohlbeleibten Femme galante mit einem in Form eines erodierten Trichters sich präsentierenden Umbilikalschanker; sie gestand ohne weiteres die direkte Kontagion durch ihren zur selben Zeit wegen Ulcus durum penis im Hospital befindlichen Amator zu. Fournier ${ }^{2}$ ) hat noch 3 weitere Primärsklerosen des Nabels beobachtet, gibt aber keine genauere Beschreibung derselben. Er erwähnt indes die bedeatsame Tatsache; daß Primäraffekte, welche annähernd in halber Höhe der Mittellinie des Thorax situiert sind, mit vierfacher Lymphdrüsenschwellung - der Achselhöhlen und der Inguinalbengen - einhergehen. Diesen Befund verzeichnete er bei zweien seiner Fälle, während in den beiden anderen die Leistendrüsen allein affiziert waren. Der eine seiner Fälle mit vier Bubonen ist ganz kursorisch von Veslin ${ }^{3}$ ) mitgeteilt und betraf einen 62 jährigen, gleichzeitig mit ausgebreitetem papulösen Syphilid und Papeln des Mundes und der Zirkumanalgegend behafteten Kärrner.

Die einzige ausführlichere Krankengeschichte über einen als Primäraffekt des Nabels ausgesprochenen Krankheitsfall stammt von Berliner $;^{*}$ ) doch ist auch hier die klinische Beschreibung der Nabelveränderungen keineswegs erschöpfend.

Ein 19jähriges chlorotisches Mädchen läßt sich, da der Bräutigam seit einigen Wochen an Sklerose und Exanthem erkrankt ist, vorsichtshalber untersuchen und wird gesund befunden. 4 Monate später kommt sie wieder wegen seit 2 Wochen bestehender Appetitlosigkeit und nervöser Depression nebst Kopfschmerzen und Schlingbeschwerden. Nun werden Plaques muqueuses ãer Mundschleimhaut sowie makulopapulöses Syphilid

1) Fournier. Les chancres extragénitaux. Paris 1897. p. 284.

2) Fournier. 1. c., p. 281 und 287.

3) Veslin. Relevé de chancres syph. extragénit. observés dans le service de la clinique des mal. cut. et syph. du 1er févr. 1888 au ler févr. 1889. Ann. de Derm. et de Syph. 1890, III. sér., I, p. 318.

4) Berliner. Seltene Lokalisation der Initialsklerose mit Bemer. kungen über die Verbreitung der Syphilis auf extragenitalem Wege. Monatsh. f. prakt. Derm. Bd. XX. 1895. p.376. 
des Stammes und der Extremitäten nebst Psoriasis palmaris et plantaris konstatiert. Der Primäraffekt konnte trotz eifrigen Suchens weder am Genitale noch im Munde noch an den Fingern gefunden werden, wohl aber in der Nabelfurche, nachdem die Kranke selbst auf eine dort seit 5 Wochen befindliche Wunde aufmerksam gemacht hatte. Letztere war $1 / 2 \mathrm{~cm}$ lang und $3 / 4 \mathrm{~cm}$ breit, schmerzlos, schmutzig grau belegt, mäßig absondernd und nicht ausgesprochen hart. Die Leistendrüsen waren nicht sehr merklich vergrößert. Wiederholte Kohabitation mit dem Bräutigam ward zugegeben, perverse Ausführung derselben jedoch von beiden Seiten geleugnet; die Kranke will sich aber gelegentlich eines vor Wochen stattgefundenen tête-à-tête mit dem Fingernagel gekratzt haben. B e r lin er läßt unentschieden, ob das Syphilisgift schon am Finger deponiert gewesen oder ob das Kratzen nur die Eingangspforte für das von der infizierten Kleidung gelieferte Virus geschaffen habe. Es bleibe dahingestellt, ob hier wirklich syphilitischer Primäraffekt oder das in der Nabelfurche nicht selten breite Kondylom vorgelegen hat. Der Nabelprimäraffekt geht, wie die vier Beobachtungen von Fournier und mein eigener Fall lehren, regelmäßig mit Schwellung der Leistendrüsen einher, wclche jedoch in Berliners Fall nicht sonderlich vergrößert waren.

Die große Seltenheit dieser Lokalisation des syphilitischen Primäraffektes, ganz besonders aber der Umstand, daß in der Literatur eine Beschreibung der hier in Frage kommenden morphologischen Veränderungen bislang fehlt, rechtfertigen es; wenn ich hier eine solche Beobachtung des genaueren mitteile:

C. B., 26 Jahre, Bäckergehilfe. Aufgenommen am 28. August 1895 in die Wiener Klinik für Syphilis:

P. gibt an, daß seine Nabelaffektion seit einem Monate, der Ausschlag seit 8 Tagen bestehe; über die Art und Weise der Übertragung ist ihm nichts bekannt.

An Stamm und Extremitäten dichtgedrängte linsen- bis halbkreuzergroße blaßlivid gerötete Eflloreszenzen, welche im Hautniveau liegen oder leicht äber dasselbe vortreten, die meisten sind mit einem zentralen einem Follikel entsprechenden kleinen Knötchen versehen. Das Exanthem ist durchwegs gleichmäßig verteilt, auch in der Sternalgegend und an den Unterschenkeln finden sich reichliche Effloreszenzen, ferner an den 
Hand- und Fußrücken. An den Handtellern halblinsengroße, mehr bräunüber das Hautnivean vortretende, mit schwieliger Epidermislag versehene, etwa hanfkorngroße Effloreszenzen. In der Skrotokruralfurche erodierte linsengroße Effloreszenzen and scharf umschriebene rotbraun glänzende flache Substanzverluste.

Inguinaldrüsen, rechts multipel bohnengroß, daneben eine größere länglich spindelförmige Drüse, links die Drüsen über haselnußgroß, derbe indolent. Kubitaldrüsen beiderseits über walnußgroß.

Nabelstumpf geschwellt, haselnuBgroß; an seiner oberfläche ein scharfrandiges, kreisförmiges, flaches Geschwür, rotbraun glänzend, zum Teile mit dünnem, fest haftenden, blabgelben Belage versehen, in der oberen Hälte mit einer Kruste bedeckt, seine Konsistenz ziem. lich derb. Das Geschwür setzt sich links obengegen die Nabelfurche fort und erscheint hier bereits bläulichweib überhäutet.

Lymphdrüsen am Rande des M. pectoralis major nicht tastbar, Axillardrüsen beiderseits über walnußgroß, hart, indolent.

Zervikaldrüsen multipel über baselnußgroß. An den Fußsoblen über linsengroße, schmutzigbraune, umschriebene Effloreszenzen. An Wangen und Stirne, den Mundwinkeln, der Kinnfurche und Nasenflügeln leicht elevierte, blabrote, mit dünner, fettiger Schuppenauflagerung versehene Effloreszenzen. Auch im Nacken lentikulär-papulöse Effloreszenzen, ferner an Kopfhaut und Ohrmuscheln und im äußeren Gehörgange.

Therapie: Täglich eine Einreibung mit Kalomelsalbe. ${ }^{1}$ )

14. Oktober: Nach 16 Kalomeleinreibungen à $3 g$ die Sklerose am Nabel noch nicht vollständig überhäutet, der Belag eingetrocknet; es besteht noch leichte Derbheit und Lividfärbung. Inguinaldrüsen nunmehr beiderseits über bohnengroß. Das Exanthem allenthalben im Hautniveau, in der Färbung jedoch ganz unverändert. Auch im Gesichte und an den Fußsohlen der Effloreszenzen noch immer deutlich hervortretend, Gaumenbögen lebhaft gerötet. Von jetzt ab Einreibungen mit Ungut. cin.

5. Oktober: Nach 16 Kalomeleinreibungen und 21 Einreibungen mit grauer Salbe Entlassung.

2. Spitalsaufenthalt 30. November 1895 bis 7. Januar 1896: Roseola anulata. Papulae mucosae oris. Inunktionen mit Ungut. bydrarg.

Es ist nicht zweifelhaft, daß in unserem Falle wirklich eine primärsyphilitische Nabelaffektion vorgelegen hat und nicht etwa eine Wund-oder Infiltratbildung anderer Provenienz, insbesondere auch keine nässende Papel. Das Aussehen der Wunde am Nabel bot das typische Bild des syphilitischen

1) Rille. Über Kalomeleinreibungen bei Syphilis. Festschrift für F. J. P i c k, Arch. f. Derm. u. Syph. Bd. XLIV. 1898. p. 111-140. 
Primäraffektes, wie es sich uns täglich an den Genitalien anderer Kranker präsentierrt und wie es besonders aus den klassischen Beschreibungen von Ric ord und Fournier seit langem bekannt ist. An der Oberfläche des durch die in Rede stehende Affektion eigenartig abgeflacht erscheinenden Nabels $\mathrm{sa \beta}$ ein linkerseits bis in die Nabelfurche reichender glatter, fast zehnpfennigstückgroßer, scharf begrenzter Substanzverlust mit der für Primärsklerose charakteristischen braunroten, infolge seröser Absonderung firnisartig glänzenden Basis, welche zom Teile mit einem nicht abstreifbaren, pseudomembranösen Belage versehen war. Schwammige Wucherung oder ranzigfötider und diphtheroider Belag, wie sich derlei bei breiten Kondylomen der Nabelgegend vorfindet, war hier nicht zu sehen. Von diagnostischer Wesenheit ist namentlich die Härte des Untergrundes, nicht allein weil sie an sich ein pathognomonisches Kennzeichen jedweden, auf seiner Akme befindlichen Primäraffektes ist, sondern weil sie auch viele Wochen hindurch persistierte und selbst 7 Wochen nach Auftreten des Exanthems noch andauernd nachweisbar war.

In diagnostischer Beziehung ist für den Primäraffekt am Nabel das Vorhandensein einer multiplen Sklera denitis gleichzeitig an vier Körperstellen von geradezu absoluter Beweiskraft. Primäraffekte, welche in halber Höhe des Stammes sitzen, können mit spezifischer indolenter Schwellung der links- oder rechtsseitigen ing u in alen und axillaren. Lymphdrüsen einhergehen je nach der Körperhälfte, auf welcher die Sklerose situiert ist. Befindet sich die primäre Manifestation überdies noch in der Medianlinie, wie z. B. am $\mathrm{Nabel}$, so kann es zur Entwicklung von vier Bubonen, je einem in jeder Leiste und in jeder Axilla kommen. Unter vier von Fournier beobachteten Fällen von Umbilikalschanker war dies zweimal der Fall, während in den beiden anderen bloß die Leistendrïsen affiziert waren. Es sind demnach die Inguinaldrüsen mit größerer Regelmäßigkeit induriert als die Achseldrüsen. Auch mein Fall zeigt das klassische Bild der vier Bubonen.

Diese klinischen Tatsachen lassen sich mit dem, was über die Verteilung der Lymphgefäße der Nabelgegend bekannt ist, wohl gut in Einklang bringen. 
Nach Hy rtl') begeben sich die Lymphgefäße der Banchwand teils in die hochliegenden Leistendrüsen, teils über die Seiten des Thorax hinauf in die Achseldrüsen. Die tiefgelegenen Lymphgefäße der Bauchwand dürften sich seiner Meinung nach vorzugsweise in jene Drüsenkette entleeren, welche die Vasa iliaca begleitet.

Nach $R a u b e r,{ }^{2}$ ) welcher auf Sappey fußt, laufen die oberflächlichen Lymphgefäße des Bauches strahlenförmig gegen die oberflächlichen Leistendrüsen, indem sie im allgemeinen den Bahnen der Vasa circumflexa ilium, epigastrica superficialia und pudenda externa folgen; $z u$ ihnen gesellen sich von der lateralen Seite her noch Lymphgefäße aus der Gesäßgegend und vom unteren Teile des Rückens. Die tiefen Lymphgefäße gelangen zum Teile an den Arteriae circumflexa ilium und epigastrica inferior zu den Glandulae inguinales; der größere Teil aber verläuft mit den Vasa lumbalia und ileo-lumbalia rückwärts, schließt hier zuweilen einige Glandulae iliacae superiores ein und dringt mit den Lymphgefäßen des Rückens hinter dem M. psoas her zur Wirbelsäule in die Lendendrüsen.

Nach neueren von Gerot $\mathbf{a}^{3}$ ) angestellten anatomischen Untersuchungen folgen die Lymphgefäße der Bauchhaut den Bahnen der Arterien und münden nach oben in die Glandulae pectorales, nach unten in die Glandulae subinguinales superficiales. Was die tiefen Lymphgefäbe des Bauches betrifft, so konnte Ge rota solche von der Nabelgegend aus füllen, welche mit den Vasa epigastrica inferiora verliefen, auf ihrem Wege mehrere kleine Lymphdrüsen durchsetzten und in den tiefen Leistendrüsen endigten. Auch in der unmittelbaren Umgebung des Nabelringes gelang es zweimal eine kleine Lymphdrüse zu injizieren. Über Lymphgefäße, die zu den Axillardrüsen ziehen, wird von Gerota zwar nichts erwähnt, es spricht aber pag. 752 .

1) H y r t l. Handb. der topograph. Anatomie. Wien 1882. Bd. I.

2) Ra u b e r. Lehrbuch der Anatomie des Menschen. 4. Aufl. von Quain-Hoffmanns Anat., Leipzig 1893. Bd. 1I., 1. Abt. pag. 222 und 225.

s) Ge rota. Über die Lymphgefäße und die Lymphdrüsen der Nabelgegend und der Harnblase. Anat. Anz. Bd. XII. 1896. p. 91. 
Merkel ${ }^{1}$ ) die Vermutung aus, daß es auch den Vasa epigastrica folgende, von der Nabelgegend aus verlaufende Lymphgefäße geben dürfte Meiner Meinung nach muß man auch mit der Möglichkeit individueller Verschiedenheiten in der Verteilung und Anastomosierung der Lymphgefäße rechnen. Daraus erklärt sich vielleicht das Fehlen der Axillardrüsenschwellung in den beiden Fällen von Fournier gegenüber deren Vorhandensein in zwei anderen Beobachtungen dieses Autors gleichwie in meinem eigenen Falle. Im Falle Berliners, welcher mir, wie bereits erwähnt, diagnostisch nicht einwandsfrei erscheint, ist auf die Axillardrüsen wohl überhaupt nicht geachtet worden.

Bemerkenswert ist es ferner, daß von anatomischer Seite die Glandulae pectorales dem Bereiche der uns hier interessierenden Lymphgefäßverteilung beigezählt werden. In meinem Falle waren aber die am Rande des Musculus pectoralis major gelegenen Lymphdrüsen überhaupt nicht zu tasten; bekanntlich partizipieren die letzteren in einzelnen Fällen von sekundärer Syphilis an der generalisierten Drüsenschwellung.

Die Existenz dieses vierfachen Primärbubos im Gefolge des Umbilikalschankers läßt sehr wohl vermuten, daß bei so proximalem Sitze des Initialaffektes - gewissermaßen im Mittelpunkte der Körperoberfläche - auch das Vordringen des syphilitischen Virus im $\mathrm{Körper}$, welches doch vorwiegend auf dem Wege der Lymphbahnen erfolgt, sich wesentlich rascher vollzielsen wird als bei irgend einer anderen, mehr distalen Lokalisation des Primäraffektes wie z. B. an Kopf oder Genitale. Es dürfte also in Fällen von $\mathrm{Nabelprimäraffekt}$ die sog. $z$ weite In $\mathrm{k}$ bation eine Abkürzung erfahren und die Proruption des Exanthemsund dersonstigen Allgemeinerschéinungen schneller von statten gehen als in den gewöhnlichen Fällen von rezenter Sy$p h i l i s$. Auch für jene seltenen Fälle, wo sich kon te mpor är an zwei oder mehreren weit von ein ander abliegenden Körperstellen Primäraffekte befinden (z. B. gleichzeitig an

1) M e r k e l. Handbuch der topogr. Anatomie. Braunschweig 1899. Bd. II. p. 429. 
Mund und Genitale oder Finger und Genitale etc.) ist meiner Ansicht nach die gleiche Beschleunigung im Auftreten der Konsekutiva zu postulieren. Diese von mir hier hervorgehobenechronologische Eventualität ist abermeines Wissens noch ronkeinem der zahlreichen Bearbeiter der extragenitalen Syphilisinfektion in Erwägung gezogen worden.

Bei dem geringen Umfange der bis jetzt vorliegenden Kasuistik läßt sich das klinische Bild der syphilitischen Initialläsion am Nabel vorläufig noch nicht konstruieren. Aus unserer Beobachtung geht bloß bezüglich des Krankheitsverlaufes hervor, daß die Dauer des primären Ulkus an dieser Stelle nicht gerade eine kurze ist, da unser Primäraffekt etwa 9 Wochen hindurch persistierte und noch in der 7. Woche seines Bestandes deutliche Derbheit erkennen ließ. Es mag dies, abgesehen von anderweitigen in der feineren anatomischen Konfiguration dieser Körpergegend begründeten Ursachen auch noch daran liegen, daß Wundbildungen der Nabelgegend infolge ihres Sitzes nicht leicht gereinigt werden können.

Über den Infektionsmodus wußte unser Patient keine Angaben zu machen. Die Ursache der am Stamme lokalisierten Primäraffekte ist, wenn man von den im Gefolge der Laktation an der Mamma, gleichwie von den durch $\mathrm{Kuß}$ oder Biß entstehenden Infektionen absieht, gewöhnlich überhaupt nicht aufzuklären. Direkte Übertragung vom Genitale her gelegentlich der sexuellen Kontamination ist für den Fall von Leloir und für den einen Fall Fourniers nachgewiesen, welche beide korpulente von primärsyphilitischen, genitalkranken Männern infizierte Frauen betrafen. Auch im Falle $\mathrm{Neu}$ manns liegt direkte Kontagion vor. Die Übertragung erfolgte hier auf ein Kind durch Zusammenschlafen mit der sekundärsyphilitischen Mutter, während in der Epidemie zu Rochechouart, welche durch eine mit Mundpapeln behaftete Hebamme verschuldet wurde, indirekte mittelbare Übertragung durch den mit syphilitischem Virus imprägnierten Finger erfolgte.

In diagnostischer Beziehung kommt neben dem Karzinom in erster Linie die sekundäre Syphilis in Frage $u$. zw. weniger in ihrer vegetierenden als breites Kon- 
dylom bekannten Form als in den mehr initialen, in Gestalt ron Erosionen, bald dunkelrot, bald grau belegt erscheinenden papulösen Infiltraten. Gewisse Ähnlichkeit kann auch eine durch Smegmaanhäufung in der Nabelfurche hervorgerufene ekzemartige Entzündung der Nabeloberfläche zeigen oder insbesondere eine lokale Eruption von $\mathrm{Pemphigus} \vee \mathrm{ulg}$ aris und vegetans, die im Initialstadium dort sogar ganz isoliert vorkommen kann. Auch P s or i a s is vulg aris kommt ev. in Betracht, da sie hier ähnlich wie an der Glans penis als schuppenlose oder fettig desquamierende, dunkelrote, bisweilen glänzende Infiltration auftritt. So habe ich einen bis dahin antiluetisch behandelten Psoriatiker gesehen, bei welchem sich die Schuppenflechte außer an Nabel und Genitale nur noch an der. Kopfhaut und an fast sämtlichen schmutziggelb verfärbten, dystrophischen Fingernägeln präsentierte.

Ob eine spezielle Form der im übrigen individuell variierenden $\mathrm{Nabeln}$ arbe eine besondere Disposition für die Haftung des primärluetischen Virus abgibt, läßt sich nicht sagen. Da besonders ein tiefer und retrahierter Nabel die Bildung von Intertrigo begünstigt, so ist es nur wahrscheinlich, daß sowohl diese Nabelform als auch Ekzem und Intertrigo des Nabels einen Locus minoris resistentiae für den syphilitischen Primäraffekt abgeben werden, zumal zur Haftung des syphilitischen Virus eine Läsion der Kontinuität die unerläßliche Voraussetzung ist. In diesem Sinne könnte auch die $\mathrm{Krätze}$, welche in der Nabelgegend nicht so selten lokalisiert ist, eine Hilfsursache der syphilitischen Infektion bilden und es sind Primäraffekte auf skabiösem Boden nicht allein am Penis (F o urnier), sondern auch in der Unterbauchgegend ( $\mathrm{Ver} \mathrm{ch}$ è $\mathrm{re}$ ) bereits konstatiert worden. ${ }^{1}$ )

') d'Aulnay. Des divers modes de contagion dans la Syphilis acquise. Journ. des mal. cut. et syph. Bd. VII. 1895. p. 487. 